\title{
ON THE "FREEZING" METHOD FOR NONLINEAR NONAUTONOMOUS SYSTEMS WITH DELAY ${ }^{1}$
}

\author{
MICHAEL I. GIL' \\ Ben Gurion University of the Negev \\ Department of Mathematics \\ PO Box 653, Beer-Sheva 84105, Israel \\ E-mail: gilmi@black.bgu.ac.il
}

(Received December, 1998; Revised January, 2000)

\begin{abstract}
Nonlinear nonautonomous differential systems with delaying argument are considered. Explicit conditions for absolute stability are derived. The proposed approach is based on the generalization of the "freezing" method for ordinary differential equations.
\end{abstract}

Key words: Nonlinear Time-Variant Retarded Systems Absolute Stability.

AMS subject classifications: $93 \mathrm{D} 05,34 \mathrm{~K} 20$.

\section{Introduction and Statement of the Main Result}

The present paper is devoted to the stability of nonlinear nonautonomous differential systems with delaying argument. Stability of systems of nonlinear differential equations with delay has been discussed by many authors, cf. [6, 8, 9], etc. The basic method for stability analysis is the direct Lyapunov method. By this method, many very strong results are obtained. But finding Lyapunov's functionals for nonautonomous retarded systems is usually difficult. In this paper, we investigate the stability of nonlinear nonautonomous differential systems with delaying argument, whose linear parts have slowly varying coefficients and whose nonlinear parts have linear majorants. Our approach is based on the extension of the "freezing" method for ordinary differential equations, which in the linear case, was developed by V.M. Alekseev [1, 10, 7] (see also [5, Section 3.2]). The method was extended to nonlinear ordinary differential equations in the paper [2]. In [4] the "freezing" method has been generalized to linear differential equations with delay.

Consider in a complex Euclidean space $C^{n}$ with the Euclidean norm $\|\cdot\|_{C^{n}}$ the equation

$$
\dot{x}(t)=A_{0}(t) x(t)+A_{1}(t) x\left(t-h_{1}\right)+\ldots+A_{m}(t) x\left(t-h_{m}\right)
$$

\footnotetext{
${ }^{1}$ This research was supported by the Israel Ministry of Science and Technology.
} 


$$
+F\left(t, x(t), x\left(t-h_{1}\right), \ldots, x\left(t-h_{m}\right)\right)(\dot{x} \equiv d x / d t ; t>0)
$$

where $h_{k}$ are positive constants, and $A_{k}(t)$ are variable $n \times n$-matrices with the properties

$$
\left\|A_{k}(t)-A_{k}(s)\right\|_{C^{n} \rightarrow C^{n}} \leq q_{k}|t-s|\left(q_{k}=\text { const } \geq 0 ; t, s \geq 0 ; k=0, \ldots, m\right)
$$

and

$$
\sup _{s \geq 0} \sum_{k=0}^{m}\left\|A_{k}(s)\right\|_{C^{n} \rightarrow C^{n}}<\infty .
$$

In addition, $F:[0, \infty) \times C^{n m} \rightarrow C^{n}$ is a continuous function satisfying the condition

$$
\begin{aligned}
& \left\|F\left(t, z_{1}, \ldots, z_{m}\right)\right\|_{C^{n}} \\
& \quad \leq \sum_{k=1}^{m} \gamma_{k}\left\|z_{k}\right\|_{C^{n}}\left(\gamma_{k}=\text { const } \geq 0 ; t \geq 0 ; z_{k} \in C^{n}, k=1, \ldots, m\right) .
\end{aligned}
$$

A solution of $(1.1)$ is an absolutely continuous function $x:[-\eta, \infty) \rightarrow C^{n}$, which satisfies this equation on $(0, \infty)$ almost everywhere with the initial condition

$$
x(t)=\Phi(t) \text { for }-\eta \leq t \leq 0,
$$

where

$$
\eta=\max _{k=1, \ldots, m} h_{k}
$$

and $\Phi(t)$ is a given, continuous vector-valued function. The existence and continuation to infinity of solutions to (1.1) for all continuous $\Phi(t)$ is assumed. For conditions for solution existence see for instance, $[6,8,9]$.

We will say that the zero solution of equation (1.1) is absolutely stable in the class of nonlinearities (1.4), if there is a positive constant $M_{0}$ independent of a concrete form of $F$ (but dependent on $q_{0}, \ldots, q_{m}$ ), such that

$$
\|x(t)\|_{C^{n}} \leq M_{0} \sup _{s \in[-\eta, 0]}\|\Phi(x)\|_{C^{n}(t \geq 0)}
$$

for any solution $x(t)$ of (1.1) with the initial condition (1.5). Let $A$ be a constant $n \times n$-matrix, and let $\lambda_{k}(A)(k=1, \ldots, n)$ denote the eigenvalues of $A$ including their multiplicities. The following quantity plays an essential role hereafter:

$$
g(A)=\left(N^{2}(A)-\sum_{k=1}^{n}\left|\lambda_{k}(A)\right|^{2}\right)^{1 / 2},
$$

where $N(A)$ is the Hilbert-Schmidt (Frobenius) norm of $A$, i.e., $N^{2}(A)=$ $\operatorname{Trace}\left(A A^{*}\right)$. The relations $g^{2}(A) \leq N^{2}(A)-\left|\operatorname{Trace} A^{2}\right|$, and 


$$
g(A) \leq \sqrt{1 / 2} N\left(A^{*}-A\right) \text { and } g\left(A e^{i \theta}+z I\right)=G(A)(\theta \in \boldsymbol{R}, z \in \boldsymbol{C})
$$

are true cf. [3, Section 1.1]. In the following $I$ denotes the unit matrix. If $A$ is a normal matrix: $A^{*} A=A A^{*}$, then $g(A)=0$. For a fixed $s \geq 0$ let $K(s, p)$ be the characteristic matrix-valued function of equation (1.1). That is,

$$
K(s, p)=B(s, p)-p I \text { where } B(s, p)=\sum_{k=0}^{m} A_{k}(s) e^{-p h} k(p \in C) .
$$

To formulate the result, set

$$
\Gamma(K(s, p))=\sum_{k=0}^{n-1} \frac{g^{k}(B(s, p))}{\sqrt{k !} d^{k+1}(K(s, p))}(p \in C),
$$

where $d(K(s, p))$ is the smallest modulus of eigenvalues of the matrix $K(s, p)$ :

$$
d(K(s, p)) \underset{k=1, \ldots, n}{=\min }\left|\lambda_{k}(K(s, p))\right| .
$$

Throughout the following it is assumed that all the zeros of $\operatorname{det} K(s, p)$ for every $s \geq 0$ belong to a half-plane $C\left(-\alpha_{0}\right) \equiv\left\{z \in C: \operatorname{Re} z \leq-\alpha_{0}\right\}$ with a positive $\alpha_{0}$. Let

$$
\tilde{q} \equiv \sum_{k=0}^{m} q_{k}, \quad \tilde{\gamma} \equiv \sum_{k=1}^{m} \gamma_{k}
$$

In addition, denote

$$
\begin{gathered}
\bar{V}_{\nu}=\nu+\sup _{s \geq 0} \sum_{k=0}^{m} e^{\nu h} k\left\|A_{k}(s)\right\|_{C}, \\
\bar{\Gamma}_{\nu} \equiv \sup _{t \geq 0} \sup _{\omega \in \boldsymbol{R}} \Gamma(K(t,-\nu+i \omega)), \text { and } \bar{a}_{\nu} \equiv 2 \sqrt{\bar{V}_{\nu} \bar{\Gamma}_{\nu}\left(\bar{V}_{\nu} \bar{\Gamma}_{\nu}+1\right)}
\end{gathered}
$$

with a non-negative number $\nu$. Now we are in a position to formulate the main result of the paper.

Theorem 1.1: Let conditions (1.2), (1.3) be satisfied. In addition, for a positive number

$$
\nu<\alpha_{0}
$$

let the inequality

$$
\bar{a}_{\nu}\left(\tilde{\gamma} \nu^{-1}+\tilde{q} \nu^{-2}\right)<1
$$

hold. Then the zero solution of equation (1.1) is absolutely stable in the class of nonlinearities (1.4).

The proofs are presented in the next section. Theorem 1.1. is exact: if (1.1) is a linear stable equation with constant matrices $A_{k}(t) \equiv A_{k}$, then $\tilde{q}_{u}=\tilde{\gamma}=0$ and condition (1.8) is always fulfilled.

Let us give an estimate for $\bar{\Gamma}_{\nu}$. Let

$$
\widetilde{g}_{\nu}(B) \equiv \sup _{s \geq 0} \sup _{\omega \in \boldsymbol{R}} g(B(s,-\nu+i \omega)) \text { and } d_{\nu}(K) \equiv \inf _{s \geq 0} \inf _{\omega \in \boldsymbol{R}} d(K(s,-\nu+i \omega)) .
$$


As it was was shown in $[4,5$, p. 170],

$$
\bar{\Gamma}_{\nu} \leq \sum_{k=0}^{n-1} \frac{\tilde{g}_{\nu}^{k}(B)}{\sqrt{k !} d_{\nu}^{k+1}(K)}
$$

and

$$
\tilde{g}_{\nu}(B) \leq \sup _{s \geq 0}\left[\sqrt{1 / 2} N\left(A_{0}(s)-A_{0}^{*}(s)\right)+\sqrt{2} \sum_{k=1}^{m} e^{\nu h} k N\left(A_{k}(s)\right)\right] .
$$

If matrix $K(t, \lambda)$ is normal for all $t \geq 0, \lambda \in C$, then $g(B(s, p)=0$ and we have the simple expression $\bar{\Gamma}_{\nu}=d_{\nu}^{-1}$.

\section{Proof of Theorem 1.1}

Set $R_{+}=[0, \infty)$. The space of all continuous functions defined on a segment $[a, b]$ with values in $C^{n}$ and the sup-norm $\|\cdot\|_{C[a, b]}$ is denoted by $C\left([a, b], C^{n}\right)$. Set

$$
\begin{gathered}
L(s) x(t) \equiv A_{0}(s) x(t)+A_{1}(s) x\left(t-h_{1}\right)+\ldots+A_{m}(s) x\left(t-h_{m}\right) \\
\left(x \in C\left(R_{+}, C^{n}\right) ; t, s \geq 0\right) .
\end{gathered}
$$

For a fixed $s \geq 0$ denote by $G_{s}(t)$ the Green function of the equation

$$
\dot{\phi}_{s}(t)=L(s) \phi_{s}(t)=0 \text {. }
$$

In addition, with the notation

$$
\psi(t)=\sup _{s \geq 0}\left\|G_{s}(t)\right\|_{C^{n}}
$$

assume that

$$
\tilde{\psi}_{0} \equiv \int_{0}^{\infty} \psi(t) d t<\infty, \tilde{\psi}_{1} \equiv \int_{0}^{\infty} t \psi(t) d t<\infty
$$

Consider the equation

$$
\dot{x}(t)-L(t) x(t)=f(t)
$$

where $f \in C\left(R_{+}, C^{n}\right)$.

Lemma 2.1: Under conditions (1.2), (1.3), let the inequality

$$
\widetilde{q} \tilde{\psi}_{1}<1
$$

hold. Then for any solution $x(t)$ of problem (2.3), (1.5) the estimate

$$
\begin{aligned}
& \left.\|x(t)\|_{C\left(R_{+}\right)}\right) \\
& \quad \leq c_{0}\|\Phi\|_{C[-\eta, 0]}+\tilde{\psi}_{0}\left(1-\tilde{\psi}_{1} \widetilde{q}\right)^{-1}\|f\|_{C\left(R_{+}\right)}\left(c_{0}=\text { const }\right)
\end{aligned}
$$


is valid.

Proof: Fix $s \geq 0$ and rewrite (2.3) in the form

$$
\dot{x}(t)-L(s) x(t)=(L(t)-L(s)) x(t)+f(t) .
$$

Setting $\pi(t, s)=(L(t)-L(s)) x(t)+f(t)$, we get

$$
\dot{x}(t)-L(s) x(t)=\pi(t, s) .
$$

A solution of the latter equation with the initial condition (1.5) can be represented as

$$
x(t)=\phi_{s}(t)+\int_{0}^{t} G_{s}(t-\tau) \pi(\tau, s) d \tau \quad(t>0)
$$

where $\phi_{s}(t)$ is the solution of the homogeneous equation (2.1) with the initial condition (1.5). Let us use the representation of solutions of homogeneous autonomous systems $[6,8]$. We can write

That representation and (1.3) give

$$
\phi_{s}(t)=G_{s}(t) \Phi(0)+\sum_{k=0}^{m} A_{k}(s) \int_{-h_{k}}^{0} G_{s}\left(t-\tau-h_{k}\right) \Phi(\tau) d \tau .
$$

$$
\left\|\phi_{s}(t)\right\|_{C^{n}} \leq c_{1}<\infty(c=\text { const; } t, s \geq 0)
$$

since the Green function is bounded according to (2.2). Moreover,

$$
c_{1} \leq c_{2}\|\Phi\|_{C\left([-\eta, 0], C^{n}\right)}\left(c_{2}=\text { const }\right) .
$$

From (2.6) the inequality

follows. According to (1.2)

$$
\|x(t)\|_{C^{n}} \leq c_{1}+\int_{0}^{t} \psi(t-\tau)\|\pi(\tau, s)\|_{C^{n}} d \tau
$$

$$
\begin{gathered}
\|\pi(\tau, s)\|_{C^{n}} \leq \sum_{k=0}^{m}\left\|\left(A_{k}(\tau)-A_{k}(s)\right) x\left(\tau-h_{k}\right)+f(t)\right\|_{C^{n}} \\
\left.\leq \sum_{k=0}^{m} q_{k}|s-\tau|\left\|x\left(\tau-h_{k}\right)\right\|_{C^{n}}+\|f\|_{C\left(R_{+}\right.}\right)^{.}
\end{gathered}
$$

Let $t=s$. Then, taking into account that

we get

$$
\int_{0}^{t} \psi(t-\tau)\|f(\tau)\|_{C} n d \tau \leq c(f) \equiv \tilde{\psi}_{0}\|f\|_{C\left(R_{+}\right)}
$$

$$
\|x(t)\|_{C^{n}} \leq c_{1}+c(f)+\int_{0}^{t} \psi(T-\tau) \sum_{k=0}^{m} q_{k}(t-\tau)\left\|x\left(\tau-h_{k}\right)\right\|_{C^{n}} d \tau
$$




$$
=c_{1}+c(f)+\sum_{k=0}^{m} q_{k} \int_{-h_{k}}^{t-h_{k}} \psi\left(t-z-h_{k}\right)\left(t-z-h_{k}\right)\|x(z)\|_{C^{n}} d z .
$$

Hence,

where

$$
\|x(t)\|_{C^{n}} \leq \sum_{k=0}^{m} q_{k} \int_{0}^{t-h_{k}} \psi\left(t-z-h_{k}\right)\left(t-z-h_{k}\right)\|x(z)\|_{C^{n}} d z+c_{3}(f),
$$

$$
c_{3}=c_{1}+c(f)+\sup _{t \geq 0} \sum_{k=0}^{m} \int_{-h_{k}}^{0} \psi\left(t-z-h_{k}\right) q_{k}\left(t-z-h_{k}\right)\|\Phi(z)\|_{C^{n}} d z .
$$

\section{Setting}

$$
m\left(x, t_{0}\right)=\max _{0 \leq t \leq t_{0}}\|x(t)\|_{C^{n}}
$$

we arrive at the relations

$$
\begin{gathered}
m\left(t_{0}\right) \leq c_{3}(f)+m\left(t_{0}\right) \sum_{k=0}^{m} q_{k} \int_{0}^{t_{0}-h_{k}} \psi\left(t_{0}-z-h_{k}\right)\left(t_{0}-z-h_{k}\right) d z \\
\leq c_{3}(f)+m\left(t_{0}\right) \tilde{q} \tilde{\psi}_{1} .
\end{gathered}
$$

But condition (2.4) implies the inequality

$$
m\left(t_{0}\right) \leq c_{3}(f)\left(1-\tilde{\psi}_{1} \tilde{q}\right)^{-1}
$$

Taking into account that $t_{0}$ is arbitrary, we arrive at the estimate

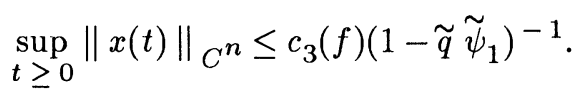

Clearly, $c_{3}(f) \leq c_{5}\|\Phi\|_{C\left([-\eta, 0], C^{n}\right)}\left(c_{5}=\right.$ const $)$. That inequality yields the result.

Corollary 2.2: Under conditions (1.2) and (1.3), let the inequality

$$
\left\|G_{s}(t)\right\|_{C^{n}} \leq C_{\nu} e^{-\nu t}(t, s \geq 0)
$$

hold with constants $\nu>0$ and $C_{\nu}$ independent of $s$. If, in addition,

$$
\tilde{q} C_{\nu}<\nu^{2}
$$

then for any solution $x(t)$ of problem (2.1), (1.5) the estimate

$$
\begin{gathered}
\left.\|x(t)\|_{C} \leq b_{\nu}\|\Phi\|_{C[-\eta, 0]}+\nu^{-1} C_{\nu}\left(1-C_{\nu} \tilde{q} \nu^{-2}\right)^{-1}\|f\|_{C(R+}\right) \\
\left(b_{\nu}=\text { const }\right)
\end{gathered}
$$

is valid.

Indeed, under condition (2.8), we easily get 


$$
\widetilde{\psi}_{0} \leq \nu^{-1} C_{\nu}, \quad \tilde{\psi}_{1} \leq C_{\nu} \nu^{-2}
$$

Now the previous lemma yields the following result.

Lemma 2.3: Let the conditions (1.2), (1.3), (2.8) and

$$
C_{\nu}\left(\tilde{\gamma} \nu^{-1}+\tilde{q} \nu^{-2}\right)<1
$$

hold. Then the zero solution of equation (1.1) is absolutely stable in the class of nonlinearities (1.4).

Proof: Condition (2.11) implies inequality (2.3) and, in addition,

$$
\tilde{\gamma} \nu^{-1} C_{\nu}\left(1-C_{\nu} \tilde{q} \nu^{-2}\right)^{-1}<1
$$

Due to (1.4) we easily get

$$
\begin{gathered}
\left\|F\left(t, x\left(t-h_{1}\right), \ldots, x\left(t-h_{m}\right)\right)\right\|_{C\left(R_{+}\right)} \leq \tilde{\gamma}\|x\|_{C[-\eta, \infty)} \\
\leq \tilde{\gamma}\left(\|x\|_{C\left(R_{+}\right)}+\|\Phi\|_{C[-\eta, 0]}\right)
\end{gathered}
$$

where $x(t)$ is the solution of (1.1). Let

$$
f(t)=F\left(t, x(t), x\left(t-h_{1}\right), \ldots, x\left(t-h_{m}\right)\right) .
$$

Then (1.1) takes the form (2.3). Now the previous corollary yields

$$
\begin{aligned}
& \|x(t)\|_{C^{n}} \leq \\
& \left.\quad b_{\nu}\|\Phi\|_{C[-\eta, 0]}+\tilde{\gamma} \nu^{-1} C_{\nu}\left(1-C_{\nu} \widetilde{q} \nu^{-2}\right)^{-1}\|x\|_{C\left(R_{+}\right)}\|\Phi\|_{C[-\eta, 0]}\right),
\end{aligned}
$$

where $x(t)$ is the solution of (1.1). Let

$$
f(t)=F\left(t, x(t), x\left(t-h_{1}\right), \ldots, x\left(t-h_{m}\right)\right) .
$$

Then (1.1) takes the form (2.3). Now the previous corollary yields

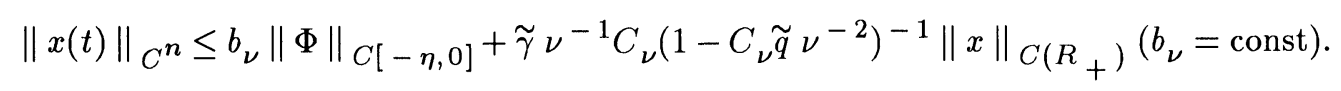

Hence, condition (2.11) implies the inequality

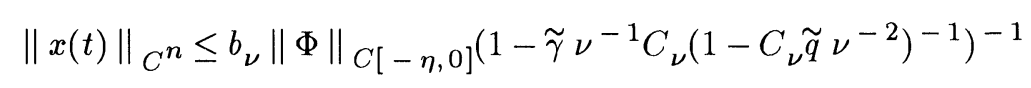

which proves the required result.

Proof of Theorem 1.1: As proved in [4, Lemma 6], the Green function of (2.1) satisfies the inequality

$$
\| G_{s}\left(t \|_{C^{n} \rightarrow C} \leq \bar{a}_{\nu} e^{-\nu t}(t, s \geq 0)\right.
$$

Now the previous lemma yields the required result. 


\section{Example}

Consider the scalar equation

$$
\begin{aligned}
\ddot{y}+(1+a(t)) \dot{y}(t) & +b(t) \dot{y}(t-1)+a(t) y(t)+b(t) y(t-1) \\
& =f(t, y(t), y(t-1))
\end{aligned}
$$

where $a(t), b(t)$ are positive functions with the properties

$$
\begin{gathered}
|\dot{a}(t)| \leq l_{0},|\dot{b}(t)| \leq l_{1},\left|a(t) \leq m_{0},\right| b(t) \mid \leq m_{1} \\
\left(l_{0}, l_{1}, m_{0}, m_{1}=\text { const }, t \geq 0\right) .
\end{gathered}
$$

In addition, the function $f:[0, \infty) \times R^{2} \rightarrow \mathbf{R}$ supplies the solvability and satisfies the condition

$$
|f(t, y, z)| \leq \gamma_{0}|y|+\gamma_{1}|z| \quad(y, z \in \mathbf{R} ; t \geq 0) .
$$

To establish stability conditions, consider the scalar equation

$$
z+a+b e^{-z}=0
$$

with positive constants $a, b$. Putting $w=z+a$, we have $w+b e^{a} e^{-w}=0$. Due to Theorem A.5 from [6, Appendix], the condition

$$
b e^{a} \leq \pi / 2
$$

guarantees that all the roots of equation $w+b e^{a} e^{-w}=0$ are in the open left halfplane. Therefore, under (3.5) all the zeros of (3.4) are in the half-plane Rez $\leq-a$. Now consider the characteristic equation of (3.1)

$$
z^{2}+\left(a+1+b e^{-z}\right) z+a+b e^{-z}=\left(z+a+b e^{-z}\right)(z+1)=0 .
$$

Under condition (3.5) we can assert that all the zeros of the latter equation are in the half-plane $R e z \leq-\alpha_{1}$ with

$$
\alpha_{1}=\min (1, a)
$$

Clearly, equation (3.1) can be rewritten in the form (1.1) with $m=1, h_{1}=1$,

$$
A_{0}(t)=\left(\begin{array}{cc}
-a(t)-1 & -a(t) \\
1 & 0
\end{array}\right) \text { and } A_{1}(t)=\left(\begin{array}{cc}
-b(t) & -b(t) \\
0 & 0
\end{array}\right)
$$

Let us assume that the condition

$$
b(t) e^{a(t)} \leq \pi / 2 \quad(t \geq 0)
$$

holds. Then according to (3.6) all the zeros of the characteristic function 


$$
\begin{gathered}
z^{2}+\left(a(t)+1+b(t) e^{-z}\right) z+a(t)+b(t) e^{-z} \\
\left(z+a(t)+b(t) e^{-z}\right)(z+1)=0
\end{gathered}
$$

for any fixed $t$ are in the half-plane

$$
\left\{z \in C: \operatorname{Re} z \leq-\alpha_{0} \equiv-\min \left\{1 \inf _{t \geq 0} a(t)\right\}\right\}
$$

Let $\nu<\alpha_{0}$, for example, $\nu=\alpha_{0} / 2$. Due to (1.6) and (3.2) we easily have,

$$
g\left(A_{0}(t)+a_{1}(t) e^{\nu-i \omega}\right) \leq g_{\nu} \equiv 1+m_{0}+m_{1} e^{\nu}(\omega \in \mathbf{R}, t \geq 0) .
$$

Moreover, (3.8) implies

$$
\lambda_{1}(K(t, p))=p+a(t)+b(t) e^{-p}, \lambda_{2}(K(t, p))=p+1
$$

Hence,

$$
\left|\lambda_{k}(K(t,-\nu+i \omega))\right| \geq \min \left\{\left|-\nu+i \omega z+a(t) b(t) e^{\nu-i \omega}\right|,|-\nu+i \omega+1|\right\} .
$$

But

$$
\begin{gathered}
\left|-\nu+i \omega+a(t) b(t) e^{\nu-i \omega}\right|^{2} \\
=\left(-\nu+a(t)+b(t) e^{\nu} \cos \omega\right)^{2}+\left(\omega+b(t) e^{\nu} \sin \omega\right)^{2} \geq\left(-\nu+a(t)-b(t) e^{\nu}\right)^{2} .
\end{gathered}
$$

So

$$
d_{\nu} \geq \min \left\{\inf _{t \geq 0}\left|a(t)-\nu-b(t) e^{\nu}\right|, 1-\nu\right\}>0
$$

since under (3.7), function

$$
a(t)-\nu+b(t) e^{-i \omega+\nu}
$$

has no zeros. According to (1.9) we get

$$
\bar{\Gamma}_{\nu} \leq M_{\nu} \equiv d_{\nu}^{-1}\left(1+d_{\nu}^{-1} g_{\nu}\right)
$$

In addition,

$$
\left\|A_{0}(t)\right\|_{C^{n} \rightarrow C^{n}} \leq 1+2 m_{0},\left\|A_{1}(t)\right\|_{C^{n} \rightarrow C_{n}} \leq m_{1}(t \geq 0) .
$$

So $\bar{V}_{\nu} \leq \bar{W}_{\nu}$, where

$$
\bar{W}_{\nu}=\nu+1+2 m_{0}+m_{1} e^{\nu} \text { and } \bar{a}_{\nu} \leq \bar{b}_{\nu}
$$

where

$$
\bar{b}_{\nu}=\sqrt{M_{\nu} \bar{W}_{\nu}\left(1+M_{\nu} \bar{W}_{\nu}\right)} .
$$


Moreover, (3.2) implies inequalities (1.2) with $q_{0}=2 l_{0}, q_{1}=2 l_{1}$. So $\tilde{q}=2\left(l_{0}+l_{1}\right)$. Condition (3.3) yields inequality (1.4). Now Theorem 1.1 implies:

Proposition 3.1: Let conditions (3.2) and (3.7) be fulfilled. In addition, for a positive $\nu<\min \left\{1, \inf _{t \geq 0^{a}} a(t)\right\}$, let the inequality

$$
2 \bar{b}_{\nu}\left[\left(\gamma_{0}+\gamma_{1}\right) \nu^{-1}+\left(l_{0}+l_{1}\right) \nu^{-2}\right]<1
$$

hold. Then the zero solution of equation (3.1) is absolutely stable in the class of nonlinearities (3.3).

\section{References}

[1] Bylov, B.F., Groman, B.M., Nemyckii, V.V. and Vinograd, R.E., The Theory of Exponents, Nauka, Moscow 1966 (in Russian).

[2] Gil', M.I., The freezing method for nonlinear equations, Diff. Eqns. 25 (1989), 912-918 (trans. from Russian).

[3] Gil', M.I., Norm Estimations for Operator-Valued Functions and Applications, Marcel Dekker, Inc., New York 1995.

[4] Gil', M.I., On the freezing method for differential equations with delay, Analysis 17 (1997), 25-33.

[5] Gil', M.I., Stability of Finite and Infinite Dimensional Systems, Kluwer Academic Publishers, Boston-Dordrecht-London 1998.

[6] Hale, J.K., Theory of Functional Differential Equations, Springer-Verlag, New York 1977.

[7] Izobov, N.A., Linear systems of ordinary differential equations, Itogi Nauki $i$ Tekhniki. Mat. Analis 12 (1974), 71-146 (in Russian).

[8] Kolmanovskii, V. and Myshkis, A., Applied Theory of Functional Differential Equations, Kluwer Academic Publishers 1992.

[9] Kolmanovskii, V. and Myshkis, A., Introduction to the Theory and Applications of Functional Differential Equations, Kluwer Academic Publishers (1998).

[10] Vinograd, R., An improved estimate in the method of freezing, Proc. AMS 89:1 (1983), 125-129. 


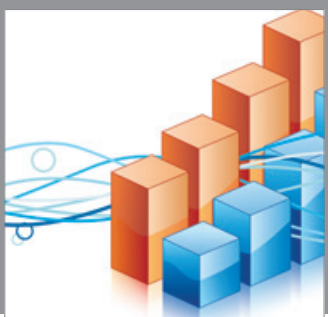

Advances in

Operations Research

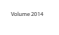

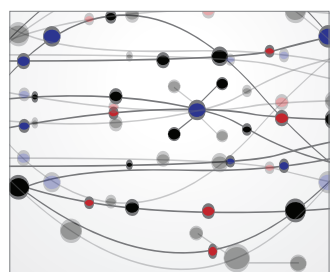

\section{The Scientific} World Journal
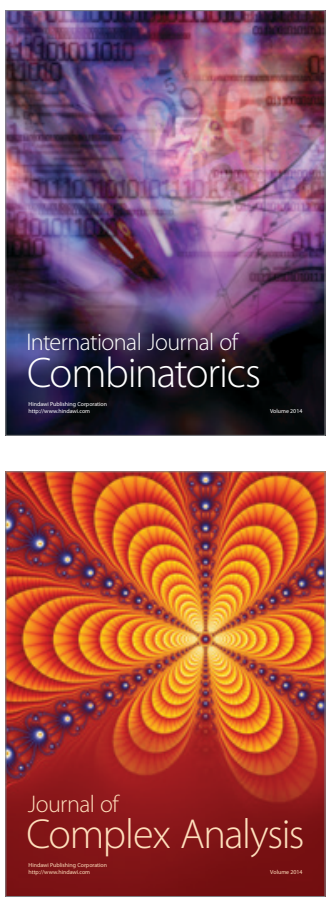

International Journal of

Mathematics and

Mathematical

Sciences
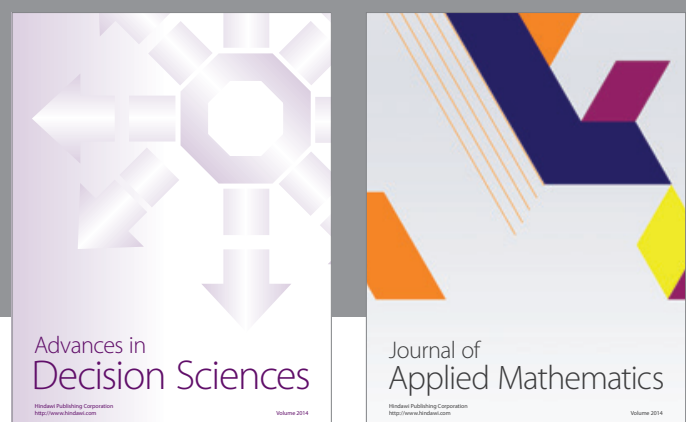

Journal of

Applied Mathematics
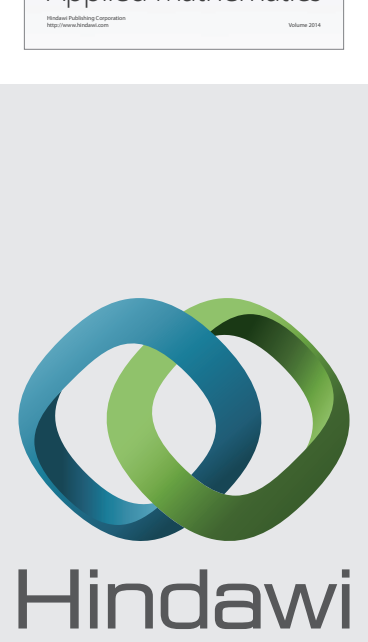

Submit your manuscripts at http://www.hindawi.com
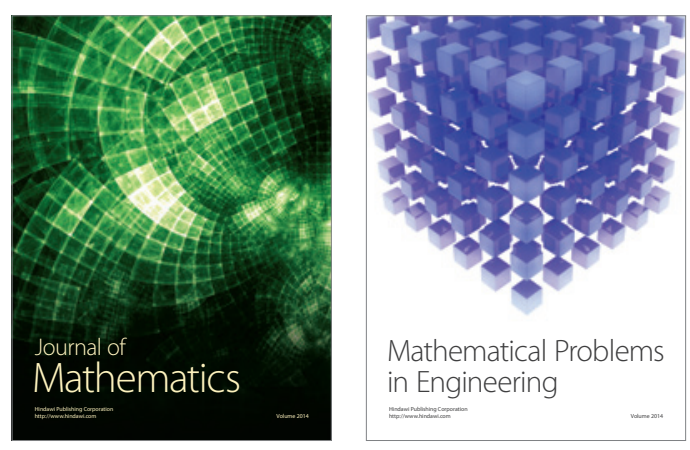

Mathematical Problems in Engineering
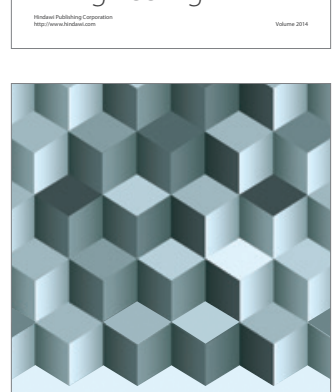

Journal of

Function Spaces
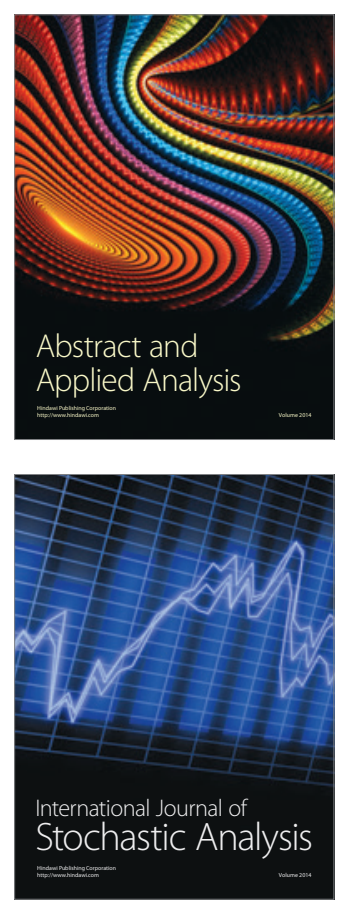

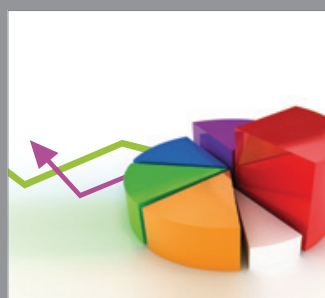

ournal of

Probability and Statistics

Promensencen
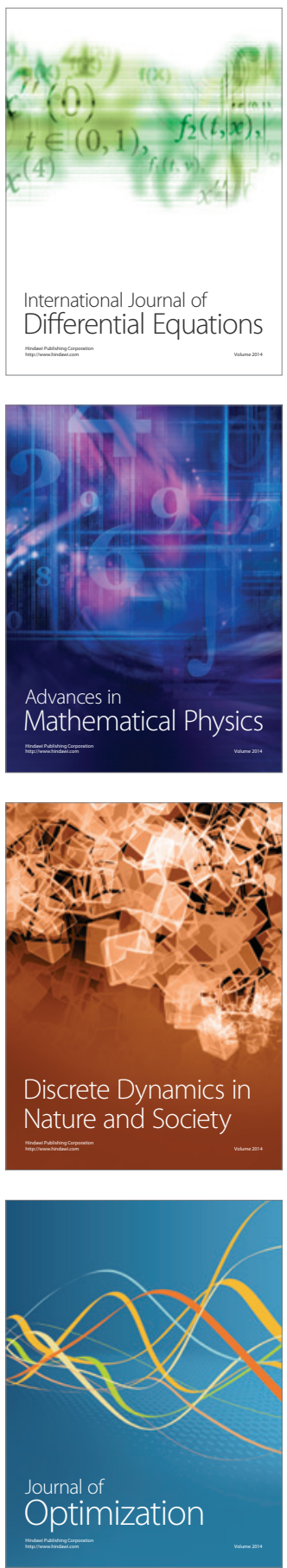